\title{
Escala de clima organizacional para organizações de saúde: desenvolvimento e estrutura fatorial
}

\section{Organizational climate scale for health organizations: development and factorial structure}

\author{
Igor Gomes MENEZES \\ Leonardo Rodrigues SAMPAIO' \\ Ana Cristina Passos GOMES \\ Flora Silva TEIXEIRA \\ Pricila de Sousa SANTOS
}

\begin{abstract}
Resumo
O clima organizacional, um dos construtos de maior complexidade na área organizacional, apresenta características que podem ser percebidas de formas distintas por diferentes profissionais. Este estudo teve como objetivo desenvolver e validar a Escala de Clima Organizacional para Organizações de Saúde. Para validar o instrumento foram investigados 668 trabalhadores da área de saúde, que responderam a 100 itens, distribuídos em 13 dimensões. Foi utilizada para estudo da validade a análise fatorial e, para exame da consistência interna, o cálculo do alpha de Cronbach. Os resultados apontaram como melhor solução fatorial a estrutura de 64 itens, dispostos em sete fatores. A consistência interna da medida foi de 0,934. A Escala de Clima Organizacional para Organizações de Saúde demonstrou boa qualidade psicométrica, considerando-se o contexto cultural investigado. Sugere-se, entretanto, a ampliação dos estudos para outros tipos de amostra e organizações, de modo a verificar a validade transcultural do construto.
\end{abstract}

Unitermos: Clima organizacional. Comportamento organizacional. Escala. Saúde. Validade de construto.

\begin{abstract}
Organizational climate, as one of the most complex constructs in the organizational area, possesses characteristics that may be perceived in distinct ways by different professionals. This study aimed to develop and validate the Organizational Climate Scale for Health Organizations. In order to validate the tool, 668 health workers were investigated, responding to 100 items, distributed across thirteen dimensions. Factorial analysis was used to validate and calculate Cronbach's alpha in order to estimate internal consistency. The results suggest the best factorial solution was a structure composed of 64 items, organized into seven factors. The internal consistency was 0.934. The Organizational Climate Scale for Health Organizations shows good psychometric quality, considering the cultural context investigated. However, it is recommended that these studies be extended to other types of samples and organizations, in order to verify the cross-cultural construct validity.
\end{abstract}

Uniterms: Organizational climate. Organizational behavior. Scale. Health. Construct validity.

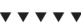

- Universidade Federal do Vale do São Francisco, Curso de Psicologia, Colegiado de Psicologia. Av. José de Sá Maniçoba, s/n., Campus Petrolina, 56304-917, Petrolina, PE, Brasil. Correspondência para/Correspondence to: I.G. MENEZES. E-mail: <igmenezes@yahoo.com.br>. 
O estudo das percepções que os trabalhadores constroem acerca de diferentes aspectos do seu trabalho e que são responsáveis por guiá-los em linhas consistentes de ação tem sido uma das temáticas de maior interesse no campo do comportamento organizacional. Diferentes são os construtos investigados pelo campo, sendo o clima organizacional um dos atributos mais relevantes à detecção dos elementos reguladores e orientadores do comportamento humano dentro de organizações.

As pesquisas sobre clima em organizações ganharam uma maior popularidade dentro da área de Administração a partir da década de 1950, nos Estados Unidos (Forehand \& Gilmer, 1964). Não obstante haja mais de meia década de estudos sobre clima organizacional, o desenvolvimento de estratégias voltadas para a investigação do construto ocorreu em maior monta somente a partir dos anos 90, ganhando maior destaque nas grandes empresas, principalmente as que já haviam desenvolvido programas de saúde do trabalhador e melhoria da qualidade de vida no trabalho (Toro, 2001).

Considerando o conjunto de métodos e técnicas adotados para o conhecimento do clima organizacional, as escalas psicométricas são um dos instrumentos mais empregados atualmente para o estudo de tal atributo, devido ao seu rigor metodológico e por serem subsidiadas por procedimentos estatísticos que conferem grande poder de análise e inferência das informações prestadas. Haja vista a pequena quantidade de medidas brasileiras desenvolvidas e validadas para avaliar o clima organizacional (Crespo, 2005; Laros \& Puente-Palacios, 2004; Martins, Oliveira, Silva, Pereira \& Sousa, 2004; Tamayo, 1999), faz-se necessária a construção de instrumentos cada vez mais específicos e competentes em avaliar diferentes fatores pertinentes a cada realidade de trabalho.

Os estudos de validação de medidas de clima organizacional, dentro do contexto nacional, ainda não contemplam um instrumento que avalie descritores de clima voltados para profissionais que trabalham em organizações de saúde e que destaquem fatores relacionados à "saúde no trabalho", "segurança no trabalho", "organização do trabalho" e "relacionamento com a comunidade". Procurando, portanto, preencher tal lacuna, assim como produzir conhecimento sobre o tema dentro da área do comportamento organizacional, este trabalho buscou desenvolver e testar a validade de construto da Escala de Clima Organizacional para Organizações de Saúde.

\section{A polissemia do construto clima organizacional}

Assim como ocorre com boa parte dos construtos no campo do comportamento organizacional, não existe um consenso entre os pesquisadores da área que permita encontrar uma definição integrada e única para clima organizacional. O conjunto de definições geralmente empregadas para caracterizar tal construto tem originado, assim, vários quadros de referência, distintas definições operacionais e técnicas de investigação, além de resultados de pesquisas muitas vezes diversificados e contraditórios.

As premissas dos estudos sobre clima organizacional podem ser encontradas nos trabalhos sobre a Teoria de Campo, Lewin (1939). Pela Teoria de Campo, as atividades psicológicas de um sujeito desenvolvem-se em uma espécie de campo psicológico, denominado pelo próprio Lewin (1939) como campo vital. A partir da introdução de tal conceito na Psicologia Social, Lewin, Lippitt e White (1939) desenvolveram um novo estudo com o intuito de verificar quais eram as condições psicológicas criadas pelos líderes de um grupo de rapazes dentro de um ambiente controlado. Introduziam-se, assim, com tal estudo, os conceitos de clima social e atmosfera social que, somente em 1958, com os trabalhos de Argyris, ganhariam o nome de clima organizacional.

No trabalho seminal de Argyris (1958) o clima organizacional, também nomeado de "caráter homeostático", foi caracterizado a partir de elementos que representam diferentes níveis de análise. Os estudos de Argyris, por um lado, trazem avanços significativos para a consecução de uma definição mais clara e abrangente de clima organizacional, ao passo que demonstram a importância de se considerarem diferentes variáveis e níveis de análise no entendimento do construto. Entretanto, Argyris vincula a noção de clima organizacional com a de cultura organizacional, promovendo, consequentemente, uma nova pulverização no conceito de clima e aumentando a dificuldade de se aceder a uma definição consensual do atributo.

Não obstante a ampla gama de definições para clima organizacional, podem-se identificar três momen- 
tos distintos em uma tentativa de síntese da produção acerca do construto. O primeiro momento, compreendido entre as décadas de 1930 e 1960, confere uma significativa ênfase no modelo organizacional, assim como na taxonomia, contexto e estrutura do clima organizacional. A preocupação, nesse período, estava dirigida para a identificação das variáveis que compunham ou constituíam o construto ou, mais especificamente, para o estudo das dimensões do clima organizacional. Um dos maiores esforços nesse primeiro período era encontrar uma justificativa conceitual que sustentasse métodos precisos e objetivos por meio dos quais se pudesse responder à seguinte pergunta: o clima organizacional pode ser conceituado como um atributo do indivíduo ou da organização?

Buscando responder a essa questão, inúmeras foram as tentativas dos teóricos da área para criar uma definição que incorporasse os diferentes elementos que supostamente caracterizavam o construto. A definição mais distintiva e representativa desse momento foi oferecida por Forehand e Gilmer (1964), que definiram clima organizacional como um conjunto de características que: a) distinguem a organização de outras organizações; b) são relativamente duradouras ao longo do tempo; e c) influenciam os comportamentos dos trabalhadores na organização. Taguiri e Litwin (1968), seguindo a mesma direção conceitual de Forehand e Gilmer (1964), identificaram o clima organizacional como uma qualidade relativamente duradoura do ambiente interno de uma organização, que: a) é experimentada pelos seus membros; b) influencia seus comportamentos; e c) pode ser descrita em termos da avaliação de um conjunto particular de características da organização. De acordo com os autores, para se conhecer o clima organizacional é necessário avaliar um conjunto de características organizacionais que incluem desde o conhecimento da estrutura organizacional até a forma como os indivíduos a percebem no dia a dia.

Em suma, o primeiro momento de investigação do clima organizacional foi notadamente marcado pela valorização do ambiente na percepção do referido atributo e pela ênfase nos elementos do ambiente que funcionam como uma espécie de "atmosfera" favorecedora da modificação dos comportamentos dos membros organizacionais. Além disso, esse momento foi importante por considerar a necessidade de se criarem métodos de investigação que avaliassem a multidimensionalidade do construto e por realçar os elementos da cultura organizacional que guardam relações conceituais diretas com o clima organizacional. Particularmente no âmago dessa relação iniciou-se o segundo momento no desenvolvimento dos estudos sobre clima organizacional.

A proximidade conceitual entre cultura organizacional e clima organizacional, que inaugurou o segundo momento, compreendido entre os anos 1970 e 1980, deve-se ao fato de a cultura organizacional estar relacionada às crenças, valores e aspectos de natureza coletiva e ideacional, compartilhada entre trabalhadores e organizações que, em última instância, são responsáveis pela manutenção de um determinado clima na organização (Hofstede, Neuijen, Ohayv \& Sanders, 1990). De acordo com Schein (1985), a cultura organizacional pode ser entendida como um conjunto de pressupostos básicos criado, desenvolvido ou descoberto em um processo de aprendizagem coletivo para lidar com os problemas socioemocionais dos membros do grupo, que permite às organizações se adaptarem às demandas externas. Dentre a pluralidade de conceitos voltados à explicação da cultura organizacional, é praticamente consensual que a cultura é formada por valores, crenças, códigos e regras que configuram as normas comportamentais nas organizações (Cooke \& Lafferty, 1989; Kroeber \& Kluckhohn, 1952; Moran \& Volkwein, 1992; Ouchi, 1981; Swartz \& Jordan, 1980; Trice \& Beyer, 1984).

Cultura é comumente definida como uma variável mais profunda do que o clima. Entretanto, enquanto a cultura de uma organização refere-se às práticas adotadas que norteiam os trabalhadores de uma empresa, concedendo-lhe uma identidade própria (Silva \& Zanelli, 2004), o clima é a atmosfera presente nas organizações, originada mediante práticas, procedimentos e recompensas percebidos diariamente pelos empregados, intimamente ligada ao comportamento dos gestores e às ações por estes recompensadas (Schneider, Salvaggio \& Subirats, 2002). Apesar das sobreposições conceituais entre os temas, é praticamente consensual que cultura e clima organizacionais se inter-relacionam.

Os limites conceituais e metodológicos entre clima e cultura somente começaram a ser nitidamente 
demarcados a partir dos anos 90 do século passado, com o crescimento de pesquisas qualitativas e quantitativas que buscaram atestar a validade discriminante dos construtos (Glisson \& James, 2002). Atualmente, é praticamente de concordância coletiva que, enquanto o clima organizacional é uma condição temporária, formada pelos significados construídos pelos indivíduos, que guiam suas decisões e ações no ambiente organizacional, a cultura organizacional cumpre um papel mais normativo, voltado ao compartilhamento de valores que fornecem uma identidade à organização e, consequentemente, favorecem uma maior capacidade adaptativa e de autodesenvolvimento interno e externo.

As tentativas de especificar e clarificar o conceito de clima organizacional, diferentemente de outros conceitos correlatos, deu início ao terceiro momento no estudo do construto, que começou nos anos 90 e se estende aos dias atuais. Esse momento tem sido fortemente marcado tanto pela busca da identificação das características que constituem o clima organizacional, como pela investigação dos elementos que não fazem parte das definições constitutiva e operacional do construto. No âmbito dessas discussões, grande confusão conceitual ocorre entre os conceitos de clima organizacional e clima psicológico. Segundo Schneider e Reichers (1983), o clima psicológico corresponde à ligação do indivíduo com seu contexto de trabalho, enquanto o clima organizacional reflete os significados compartilhados pelas pessoas, ligados a um aspecto particular do trabalho. Embora, como afirma Martins (2000), não seja possível reduzir o clima organizacional a uma só unidade de análise, já que o mesmo depende também das interações individuais, é necessário reconhecer que o clima organizacional está mais diretamente relacionado a elementos cognitivos, ao passo que o clima psicológico encontra-se associado, em maior intensidade, com elementos afetivos do ambiente de trabalho. De fato, um conhecimento mais pormenorizado do contexto organizacional deveria contemplar avaliações multidimensionais das relações entre indivíduos e organizações (Glick, 1985).

A consecução de estudos para tornar o conceito de clima organizacional mais claro e específico foi paralelamente seguida pelo desenvolvimento de métodos e técnicas que viabilizassem a determinação dos elementos mais representativos e constituintes do construto e que permitissem medi-lo com um grau máximo de precisão. Perseguindo, assim, um crescente rigor metodológico para a investigação do clima organizacional, várias escalas psicométricas foram construídas para avaliar o construto.

Dentre as técnicas quantitativas e qualitativas empregadas para a avaliação do clima organizacional, as escalas psicométricas figuram como a técnica mais utilizada nas pesquisas sobre o tema. Diferentes foram as escalas clássica e modernamente desenvolvidas para a mensuração do construto em âmbito internacional (Friedlander \& Margulies, 1969; Hellriegel \& Slocum, 1974; House \& Rizzo, 1972; Litwin \& Stringer, 1968; Payne \& Pheysey, 1971; Pritchard \& Karasick, 1973; Schneider \& Bartlett, 1970; Toro, 2001) e nacional (Crespo, 2005; Laros \& Puente-Palacios, 2004; Martins et al., 2004; Tamayo, 1999). No entanto, até o presente momento não existe uma medida de clima organizacional que tenha sido desenvolvida e validada com o intuito de detectar as demandas de profissionais que trabalham em organizações de saúde. Buscando, assim, considerar a multidimensionalidade do construto clima organizacional na construção de uma medida adequada a esta área, este trabalho teve por escopo apresentar os estudos da estrutura fatorial da Escala de Clima Organizacional para Organizações de Saúde e contribuir com os conhecimentos teóricos e empíricos sobre um dos temas de maior centralidade no campo do comportamento organizacional.

\section{Método}

\section{Participantes}

Para o conhecimento das propriedades psicométricas da escala foram selecionados 668 trabalhadores da Secretaria de Saúde da prefeitura de uma cidade do interior da Bahia. Ao todo, foram investigados 190 setores organizacionais e 137 ocupações, considerando todas as unidades da região investigada. Foi considerado setor organizacional todo e qualquer tipo de espaço de trabalho em que o profissional de saúde desenvolve suas atividades. Dos setores organizacionais mais representativos, havia 15,5\% de centros de saúde, 10,2\% de órgãos ligados à administração, 6,9\% de hospitais e 5,4\% de unidades básicas de saúde, incluindo o Programa Saúde da Família (PSF). 
Considerando as ocupações investigadas, 18,7\% da amostra compuseram-se de técnicos auxiliares de enfermagem, 10,5\% de agentes de saúde, 6,9\% de médicos, 5,3\% de recepcionistas, 5,3\% de auxiliares de serviços gerais e 4,3\% de coordenadores de órgãos administrativos. Do total de 668 sujeitos pesquisados, 160 (24,3\%) eram do sexo masculino, 498 (75,7\%) do sexo feminino e nove sujeitos não se identificaram. Aproximadamente $44,3 \%$ dos indivíduos pesquisados encontravam-se na faixa etária entre 26 e 40 anos, 48,2\% possuíam nível médio completo e 20,2\%, nível superior. A pesquisa teve acesso a todos os membros da Secretaria de Saúde e foi censitária a estratégia de amostragem adotada.

O desenvolvimento da Escala de Clima Organizacional para avaliar profissionais de saúde surgiu a partir de uma demanda da Secretaria de Saúde de um município do Estado da Bahia. Não obstante a expressiva quantidade de instrumentos para a mensuração do clima organizacional em diferentes empresas de distintos setores da economia, até o momento não tinha sido desenvolvido um instrumento específico para avaliar as condições a que os trabalhadores que atuam nas organizações de saúde estão submetidos e, consequentemente, os diferentes níveis de satisfação que porventura se originam das percepções de sua realidade de trabalho.

Tendo em vista tal demanda e complexidade, a Escala de Clima Organizacional para Organizações de Saúde foi concebida objetivando contemplar tanto aspectos da estrutura organizacional quanto dos processos organizativos de trabalho, perpassando por temas como relacionamento interpessoal e saúde do trabalhador. Assim, 13 dimensões foram escolhidas como as mais representativas para a avaliação do clima organizacional, para as quais 100 itens foram construídos: 1) relação com o trabalho (7 itens); 2) saúde no trabalho (9 itens); 3 ) segurança no trabalho (5 itens); 4) relacionamento e espírito de equipe (11 itens); 5) comunicação (7 itens); 6) liderança (15 itens); 7) reconhecimento e recompensa ( 4 itens); 8 ) remuneração (5 itens); 9) benefícios ( 2 itens); 10) desenvolvimento profissional (13 itens); 11) organização do trabalho (7 itens); 12) estratégia (6 itens); e 13) relação com a comunidade (9itens).

Dos 100 itens desenvolvidos, oito foram elaborados de forma invertida, seguindo-se as recomendações de Pasquali (1998) sobre a importância de se evitar o erro da resposta estereotipada à esquerda ou à direita da escala de resposta. Seis outros itens foram acrescentados ao instrumento com o intuito de avaliar a percepção da importância da pesquisa por parte dos trabalhadores, assim como o seu potencial nível de aceitação. O principal objetivo da inclusão de tais itens foi verificar a potencial seriedade nas respostas dos sujeitos, dado que, caso houvesse uma baixa percepção geral de importância, as respostas aos demais itens da escala poderiam ter sido oferecidas sem o devido compromisso com os resultados da pesquisa, enviesando, assim, a interpretação das informações sobre o construto avaliado. Embora presentes no mesmo questionário de clima organizacional, tais itens não configuram uma dimensão do construto e, portanto, não foram utilizados para a investigação da estrutura fatorial da escala.

A distribuição da quantidade de itens foi realizada considerando-se a complexidade teórica do construto, que pode ser apreciada pelo número de descritores que deveria representar teoricamente cada dimensão. Destarte, foram consideradas as opiniões dos representantes da Secretaria de Saúde acerca de características do cotidiano e das realidades de trabalho que se mostrariam significativas para o julgamento dos trabaIhadores sobre aspectos do clima organizacional. No âmbito do processo de avaliação dos itens, tais representantes serviram como juízes na avaliação do conteúdo da escala.

Para mensurar a intensidade da satisfação em relação a cada item foi utilizada a escala graduada do tipo Likert, com cinco intervalos de resposta, que variavam do extremo grau de concordância ao grau máximo de discordância. Adicionalmente aos itens do instrumento de clima, foi aplicado um questionário para investigação das características pessoais e profissionais, tais como: sexo, idade, estado civil, tempo de serviço, unidade, cargo, função de chefia, setores em que já atuou, investimento em treinamento e qualificação profissional. Além de comporem um perfil geral da amostra de validação, tais variáveis foram utilizadas para a realização de estatísticas descritivas que permitissem uma avaliação do clima organizacional por unidades e ocupações. 


\section{Procedimentos}

Como se tratou de uma pesquisa de caráter censitário foi adotada uma estratégia de coleta de dados que possibilitasse o acesso aos trabalhadores em todos os turnos de trabalho. Para tanto, doze agentes de pesquisa se revezaram nos três turnos de trabalho, em função da disponibilidade de tempo de cada respondente. Com o intuito de garantir a boa qualidade das informações prestadas, além de uma quantidade de respondentes que fizesse jus a uma pesquisa censitária, o instrumento não foi do tipo autoadministrado: seus itens foram diretamente verbalizados pelo agente de pesquisa. Os critérios de seleção adotados para a seleção dos agentes de pesquisa foram: 1) apresentar escolaridade de, no mínimo, nível médio completo; e 2) ter experiência em coleta de dados em pesquisas desenvolvidas, de preferência, por institutos de pesquisas de mercado, mídia e opinião. Um treinamento prévio foi assim realizado com os agentes de pesquisa, os quais foram capacitados a não explicar ou interpretar os itens da escala, de modo a garantir as condições de padronização da medida.

Embora a pesquisa não tenha sido de caráter obrigatório, foi exposta a todos os participantes a importância da contribuição voluntária e solícita para o conhecimento da realidade do clima organizacional da Secretaria de Saúde.

Esta pesquisa foi realizada a partir de uma consultoria prestada à Secretaria de Saúde de um município situado no Estado da Bahia, que solicitou a realização de uma pesquisa de clima organizacional para todos os profissionais da área de saúde do município. Como não se tratou, a princípio, de uma pesquisa de caráter acadêmico, mas estritamente empresarial, o instrumento de pesquisa não foi submetido a um comitê de ética. Vale ressaltar que a pesquisa assumiu um delineamento de pesquisa não experimental (correlacional) e que, consequentemente, não expôs os respondentes a tratamentos experimentais nem, portanto, colocou-os em situação de perigo ou qualquer espécie de constrangimento. Aos sujeitos era facultativa a participação na pesquisa, embora a adesão tenha sido quase integral. Os dados foram analisados no âmbito externo à Secretaria de Saúde, que não teve acesso às informações individuais participantes. Não houve necessidade de identificação de nome ou qualquer tipo de informação que pusesse em risco a identidade dos sujeitos e, consequentemente, a credibilidade da investigação.

Foi entregue um Termo de Consentimento Livre e Esclarecido (TCLE) para cada participante da pesquisa. Todos os procedimentos adotados nesta pesquisa seguiram as orientações éticas previstas na Resolução 196/96 do Conselho Nacional de Saúde (BRASIL, 1996) e na Resolução 016/2000 do Conselho Federal de Psicologia- CFP (2000).

Para o estudo da validade do construto clima organizacional foi realizada uma análise fatorial exploratória dos 100 itens que compõem a medida, buscando-se verificar se o modelo teórico de 13 fatores apresentava sustentação empírica. Para tanto, utilizou-se, para a extração da análise dos fatores, a Análise dos Componentes Principais (ACP), indicado quando se deseja obter uma combinação linear não correlata das combinações das variáveis mensuradas, ou seja, quando se assume que os componentes não são correlacionados (Malhotra, 2001).

Após o estudo da estrutura fatorial da escala, procedeu-se a uma investigação dos níveis de fidedignidade dos fatores e da escala como um todo. Como a escala adotada foi do tipo Likert, que caracteriza os itens como politômicos, foi calculado o alpha de Cronbach para exame da consistência interna da medida.

\section{Resultados e Discussão}

Antes da realização das análises psicométricas, foi verificado o grau de aceitação do instrumento a partir de uma média realizada entre os seis itens de avaliação da pesquisa. Ao ser encontrada uma média (M)de 4,80 (desvio-padrão - DP=0,41), em uma escala de concordância tipo Likert que varia de 1 (discordo totalmente) a 5 (concordo totalmente), constatou-se uma elevada aceitação da realização da pesquisa, pelo que se infere um maior nível de seriedade das respostas dos participantes. Uma vez examinada a aceitação da pesquisa, procedeu-se à recodificação dos oito itens desenvolvidos de modo invertido, de modo que o valor 1 
(discordo totalmente) assumisse o valor 5 (concordo totalmente), 2 (discordo levemente) adquirisse o valor 4 (concordo levemente), e assim sucessivamente. Tal procedimento teve como objetivo posicionar todos os itens da escala de forma favorável, sendo avaliados somente a partir de uma conotação positiva. Com isso, buscou-se favorecer o estudo da dimensionalidade do construto unicamente pelo polo positivo de análise, tornando-se satisfatórias para fins de análise fatorial somente as cargas fatoriais positivas.

Após realizados tais procedimentos, buscou-se investigar a validade de construto da escala empregando-se a técnica da análise fatorial. Para tanto, o primeiro passo adotado para o estudo da análise fatorial foi verificar se a matriz de intercorrelações era fatorizável. Ao serem encontradas elevadas correlações positivas entre os itens pertencentes a um mesmo fator, além de grande parte dos níveis de significância não superiores a 0,100, de acordo com o critério adotado por Hair, Anderson, Tatham e Black (1998), verificou-se que o valor do determinante da matriz foi diferente de zero ( $D=0,00927)$, indicando a possibilidade de inversão da matriz. Uma vez tendo se certificado da possibilidade de inversão da matriz de intercorrelações, verificou-se que o valor do Teste de Adequação da Amostra Kaiser-Meyer-Olkin (KMO) foi de 0,906, o que demonstra que o método de análise fatorial é bastante adequado para o tratamento dos dados. Ao ser escolhida a análise de componentes principais como método de extração dos fatores, constatou-se que uma solução de nove componentes seria a mais indicada para avaliar o construto clima organizacional, o que corresponde a, aproximadamente, 45,2\% da variância explicada.

Sabendo-se que é bastante comum a ocorrência de uma grande saturação dos itens em diferentes fatores e que tal resultado raramente implica fatores que podem ser interpretados, escolheu-se um método de rotação fatorial para melhorar a interpretabilidade da análise fatorial. Do ponto de vista teórico, os fatores de clima são independentes e, portanto, não correlacionados, foi empregado o tipo de rotação ortogonal. Optou-se, assim, pelo uso da rotação varimax, método que distancia as cargas fatoriais elevadas das cargas baixas, tornando a saturação item-fator mais clara.

Dos 100 itens inicialmente desenvolvidos para o estudo do construto, 70 mostraram-se particularmente válidos para a avaliação do atributo. Os 30\% restantes foram eliminados tendo por base os seguintes critérios (Gorsuch, 1983; Smith \& McCarthy, 1995; Tabachnik \& Fidell, 1996): 1) o valor absoluto da carga fatorial principal do item foi menor do que 0,30 em qualquer um dos fatores ( 3 itens) ou assumiu valores negativos ( 4 itens); 2) houve cargas fatoriais similares em dois ou mais fatores em um mesmo item, com a diferença entre valores absolutos das cargas fatoriais dos itens menor do que 0,10 (8 itens); e 3) houve ausência de similaridade entre o conteúdo do item e o domínio teórico do construto (15 itens).

Considerando os 70 itens resultantes da análise fatorial (Tabelas 1, 2, 3 e 4), as dimensões de clima organizacional encontram-se dispostas na seguinte ordem: 1) liderança; 2) desenvolvimento profissional; 3) relacionamento e espírito de equipe; 4) relação com a comunidade; 5) segurança no trabalho; 6) estratégia; 7) remuneração; 8) relação com o trabalho; e 9) saúde no trabalho.

Os dois itens desenvolvidos para avaliar o fator benefícios, embora sejam específicos do contexto da organização, não apresentaram saturação com o seu fator. Um dos itens apresentou um moderado nível de correlação com a dimensão remuneração, e o segundo item mostrou-se correlacionado com um fator disperso, que reuniu distintos itens de outros fatores, mas não apresentou significado teórico. Dois itens do fator reconhecimento e recompensa apresentaram elevadas cargas fatoriais no fator liderança, o que se justifica pela proximidade no conteúdo dos itens avaliados, além da proximidade física do primeiro fator com o segundo, o que pode ter aumentado a probabilidade de relacionamento entre ambos os fatores. Como alguns descritores da dimensão comunicação apresentaram interfaces conceituais com outras dimensões, tais como liderança e relacionamento e espírito de equipe, verificou-se a presença de alguns itens com cargas fatoriais elevadas nesses fatores. Assim, decidiu-se pela manutenção do item na dimensão que tenha apresentado elevada saturação item-fator, considerando a relação teórica que cada um deles guardava com a respectiva dimensão. 0 fator organização do trabalho possuiu itens com cargas fatoriais dispersas em outros fatores não significativos e sem interfaces com tal dimensão, além de três itens que apresentaram valores de carga fatorial inferiores a 0,30 . 
Tabela 1. Fatores e saturação dos itens ( $n=17)$ da escala de clima organizacional após rotação ortogonal (Fator 1). Bahia, 2005.

\begin{tabular}{|c|c|}
\hline Itens & F1 \\
\hline Meu chefe trata de forma justa e adequada seus servidores & 0,744 \\
\hline Confio nas decisões tomadas pelo meu chefe & 0,730 \\
\hline Meu chefe tem capacidade de motivar a equipe & 0,725 \\
\hline Meu chefe aceita sugestões para melhoria do trabalho & 0,689 \\
\hline Meu chefe encara os erros não propositais como oportunidade de crescimento & 0,667 \\
\hline Meu chefe conhece bem os aspectos técnicos do seu trabalho & 0,666 \\
\hline Meu chefe me mantém informado sobre as metas da unidade & 0,654 \\
\hline Recebo elogios do meu chefe pela qualidade do meu trabalho & 0,653 \\
\hline Meu chefe tem uma visão clara sobre para onde estamos indo e como fazer para chegar lá & 0,646 \\
\hline Participo das decisões que atingem o meu trabalho & 0,614 \\
\hline A comunicação com meu chefe é fácil e aberta & 0,607 \\
\hline Sou informado sobre a contribuição do meu trabalho para os resultados da minha unidade & 0,594 \\
\hline Existe igualdade de tratamento entre chefia e subordinados & 0,576 \\
\hline Sou avaliado de acordo com os resultados do meu trabalho & 0,578 \\
\hline Sou informado pelo meu chefe sobre os assuntos relativos à unidade na qual trabalho & 0,555 \\
\hline $\begin{array}{l}\text { A chefia envolve as pessoas em decisões que afetam os diferentes aspectos do trabalho (ambiente de trabalho, } \\
\text { segurança, remuneração, estratégias etc.) }\end{array}$ & 0,520 \\
\hline Considero que os servidores se sentem livres para expressar abertamente suas opiniões & 0,349 \\
\hline
\end{tabular}

Tabela 2. Fatores e saturação dos itens ( $n=20)$ da escala de clima organizacional após rotação ortogonal (Fatores 2 e 3). Bahia, 2005.

\begin{tabular}{|c|c|c|}
\hline Itens & $\mathrm{F} 2$ & $\mathrm{~F} 3$ \\
\hline O que tenho aprendido nos treinamentos oferecidos na unidade melhora minhas condições de trabalho & 0,868 & \\
\hline O que tenho aprendido nos treinamentos oferecidos pela Secretaria melhora minha produtividade & 0,854 & \\
\hline $\begin{array}{l}\text { Os programas de treinamento e desenvolvimento têm procurado desenvolver o potencial técnico dos servidores da } \\
\text { Secretaria }\end{array}$ & 0,852 & \\
\hline $\begin{array}{l}\text { Os programas de treinamento e desenvolvimento têm procurado desenvolver o potencial humano dos servidores da } \\
\text { Secretaria }\end{array}$ & 0,821 & \\
\hline Os últimos treinamentos que recebi pela secretaria atenderam às minhas expectativas & 0,813 & \\
\hline Os conhecimentos que adquiro em programas de treinamento e desenvolvimento são aplicados no meu trabalho & 0,791 & \\
\hline Participo do levantamento de minhas necessidades de treinamento e desenvolvimento & 0,747 & \\
\hline $\begin{array}{l}\text { Os programas de treinamento e desenvolvimento preparam realmente o profissional para desempenhar as atividades } \\
\text { do cargo que ocupa }\end{array}$ & 0,733 & \\
\hline Os treinamentos preparam o indivíduo para adaptar-se à sua unidade & 0,719 & \\
\hline Na minha unidade existe plano de treinamento e desenvolvimento para todos os servidores & 0,704 & \\
\hline Realizo o meu plano de treinamento e desenvolvimento de forma negociada & 0,666 & \\
\hline O trabalho da minha equipe é bem distribuído entre os servidores & & 0,317 \\
\hline Sou acolhido como um importante membro da minha equipe de trabalho & & 0,725 \\
\hline Na minha equipe existe cooperação para o alcance das metas & & 0,695 \\
\hline Eu confio nas pessoas da equipe em que trabalho & & 0,683 \\
\hline No meu grupo de trabalho, as pessoas possuem um bom relacionamento & & 0,666 \\
\hline Sou respeitado pela minha equipe de trabalho & & 0,672 \\
\hline Na minha equipe tenho condições de ensinar o que sei aos meus colegas e aprender com eles & & 0,618 \\
\hline No meu grupo de trabalho existe liberdade de falar, criticar, sugerir e trocar ideias & & 0,576 \\
\hline Existe um forte sentido de trabalho em equipe dentro da minha unidade & & 0,527 \\
\hline
\end{tabular}


Tabela 3. Fatores e saturação dos itens ( $n=22)$ da escala de clima organizacional após rotação ortogonal (Fatores 4, 5 e 6). Bahia, 2005.

\begin{tabular}{|c|c|c|c|}
\hline Itens & F4 & F5 & F6 \\
\hline O hospital tem uma boa imagem dentro da comunidade & 0,708 & & \\
\hline A Secretaria tem uma boa imagem dentro da comunidade & 0,679 & & \\
\hline Na Secretaria existe um sistema eficaz para resolver as reclamações da comunidade & 0,670 & & \\
\hline A Secretaria comunica adequadamente à comunidade os seus projetos, investimentos e realizações & 0,609 & & \\
\hline $\begin{array}{l}\text { Existe um bom relacionamento entre os servidores e as pessoas que fazem uso dos serviços oferecidos pela } \\
\text { secretaria }\end{array}$ & 0,598 & & \\
\hline Acredito que a comunidade esteja satisfeita com a qualidade dos serviços oferecidos pela Secretaria & 0,597 & & \\
\hline Os servidores da Secretaria atendem à comunidade adequadamente & 0,556 & & \\
\hline Os usuários dos serviços de saúde reconhecem o trabalho dos servidores da Secretaria & 0,440 & & \\
\hline As condições do meu local de trabalho são satisfatórias & & 0,718 & \\
\hline Na minha unidade existem recursos de trabalho adequados para a realização do meu trabalho & & 0,655 & \\
\hline A Secretaria se preocupa com a minha segurança no trabalho & & 0,646 & \\
\hline Existem medidas de segurança adequadas para a execução do meu trabalho & & 0,615 & \\
\hline Os equipamentos de trabalho são adequados para garantir a segurança do servidor & & 0,593 & \\
\hline A Secretaria se preocupa com meu bem-estar no trabalho & & 0,559 & \\
\hline Tenho as condições necessárias para a realização do meu trabalho & & 0,438 & \\
\hline Conheço o planejamento estratégico da Secretaria & & & 0,690 \\
\hline Conheço os indicadores e as metas traçadas pela Secretaria & & & 0,676 \\
\hline Conheço a estrutura organizacional da Secretaria & & & 0,605 \\
\hline Participei, de alguma forma, do processo de elaboração do planejamento estratégico & & & 0,573 \\
\hline Consigo aplicar nas minhas atividades diárias as diretrizes do planejamento estratégico & & & 0,549 \\
\hline A Secretaria tem realizado as mudanças necessárias visando ao seu crescimento e desenvolvimento & & & 0,526 \\
\hline Na Secretaria, os servidores são estimulados a participar das contínuas mudanças & & & 0,525 \\
\hline
\end{tabular}

Tabela 4. Fatores e saturação dos itens ( $n=11)$ da escala de clima organizacional após rotação ortogonal (Fatores 7, 8 e 9). Bahia, 2005.

\begin{tabular}{|c|c|c|c|}
\hline Itens & F7 & F8 & F9 \\
\hline Minha remuneração é compatível com as responsabilidades do trabalho que realizo & 0,796 & & \\
\hline Minha remuneração está de acordo com a praticada pelo mercado & 0,796 & & \\
\hline De um modo geral, estou satisfeito com a minha remuneração & 0,789 & & \\
\hline Considero as políticas de remuneração da prefeitura justas e motivadoras & 0,685 & & \\
\hline A minha remuneração é melhor do que a da maior parte dos meus colegas de trabalho & 0,670 & & \\
\hline Meu trabalho permite que eu seja eu mesmo & & 0,340 & \\
\hline O trabalho que faço não me permite usar meus conhecimentos & & 0,327 & \\
\hline Tenho conhecimento da importância de cada tarefa que realizo & & 0,316 & \\
\hline Tenho apresentado sintomas de nervosismo, preocupação, tristeza, tensão etc. por causa do meu dia de trabalho & & & 0,682 \\
\hline $\begin{array}{l}\text { Tenho apresentado sintomas de perda de sono, dor de cabeça, dor de barriga, tremor nas mãos etc. por causa do } \\
\text { meu dia de trabalho }\end{array}$ & & & 0,657 \\
\hline O trabalho que realizo prejudica minhas relações com a comunidade, família, lazer etc. & & & 0,373 \\
\hline
\end{tabular}

Uma vez garantida a validade de construto do instrumento pelo método da análise fatorial, procedeu-se ao exame da fidedignidade das dimensões e da escala como um todo, pelo cálculo do coeficiente alpha de Cronbach. Considerando que o alpha não deve apresentar um número inferior a 0,70, o que mostraria um baixo nível de consistência interna entre os itens de uma mesma dimensão, sete dos nove fatores avaliados 
exibiram valores satisfatórios: 1$)$ segurança $(\alpha=0,772)$; 2$)$ estratégia $(\alpha=0,812)$; 3$)$ remuneração $(\alpha=0,834)$; 4$)$ relacionamento e espírito de equipe $(\alpha=0,837)$; 5 ) relação com a comunidade $(\alpha=0,839) ; 6)$ liderança $(\alpha=0,872)$; e 7) desenvolvimento profissional $(\alpha=0,0,934)$. As dimensões relação com o trabalho e saúde no trabalho apresentaram valores de confiabilidade inferiores a 0,70 $(\alpha=0,089$ e $\alpha=0,624)$.

Sabendo-se que todos os valores de correlação item-total do fator relação com o trabalho mostraram-se inferiores a 0,20, o que apontaria para um baixo poder de discriminação dos itens, segundo critérios estabelecidos pela American Institute for Research (AIR) e pela Educational Tests Services (ETS), e considerando que a deleção de qualquer dos itens não melhoraria o valor do alpha de Cronbach, optou-se pela exclusão geral de tal fator. Da mesma forma, o fator saúde no trabalho foi excluído do modelo final de validação. Duas razões justificam tal exclusão: em primeiro lugar, há a possibilidade de melhoria do alpha caso fosse deletado um dos três itens do fator. No entanto, de acordo com Pasquali (1999) não é recomendável representar um fator com menos de três itens, dada a pequena variabilidade explicativa do atributo investigado. Além disso, este fator apresentou um problema teórico de concepção que só veio a ser detectado na fase das análises psicométricas. Idealmente, os itens do fator saúde no trabalho foram desenvolvidos para avaliar a percepção do trabaIhador sobre as condições de trabalho que potencialmente impactariam na sua saúde. No entanto, três dos nove itens que, a princípio, pertenciam à dimensão, não estavam avaliando a percepção sobre condições de saúde, mas remetiam-se à avaliação da própria saúde do sujeito. Tal fato, ao afetar a unidimensionalidade do fator, promoveu um baixo nível de consistência interna entre os itens, prejudicando a adequabilidade dos parâmetros de validade e de precisão.

A estrutura final da escala de clima organizacional ficou composta por 64 itens, distribuídos em sete fatores, apresentando um valor geral de confiabilidade de 0,934.

\section{Considerações Finais}

Devido à abrangência das características investigadas, a realização de pesquisas de clima organi- zacional tem sido uma prática constante em organizações de médio e grande porte. Vários instrumentos vêm sendo desenvolvidos para avaliar o construto para diferentes tipos de organização; no entanto, poucos ainda são os instrumentos desenvolvidos com base em um aprofundamento teórico sobre os elementos que constituem o clima organizacional, e mais raros ainda são os estudos que buscam verificar se tais instrumentos apresentam validade relacionada a construto. Investigar o clima organizacional carece de uma criteriosa análise das características organizacionais mais relevantes a serem avaliadas pelos sujeitos. Logo, a dimensionalidade do construto pode exibir uma estrutura conceitual mais ou menos ampla, a depender do modo como se configura o contexto da organização e, consequentemente, como esse contexto é percebido pelos membros que dela fazem parte.

Tendo em vista a complexidade da investigação do clima organizacional e a dificuldade de se encontrarem definições operacionais consensuais para o construto, ampliar a quantidade de pesquisas voltadas ao estudo do clima é premente para o aperfeiçoamento dos conhecimentos sobre o tema. Uma das grandes dificuldades que vêm marcando a história dos estudos sobre clima é o desenvolvimento de medidas globais de avaliação capazes de detectar um amplo rol de percepções de diferentes tipos de trabalhadores, e que contemplem ainda a observação de organizações de distintos setores da economia. De certa forma, encontrar uma medida consensual e representativa das características culturais, sociais, econômicas e políticas de várias organizações é uma tarefa inviável e que demandaria grande capacidade de abstração dos elementos subjetivos de cada organização. Assim, faz-se necessário desenvolver medidas para públicos cada vez mais específicos, de modo a aperfeiçoar e validar instrumentos para organizações de distintos setores da economia e que prestam diferentes tipos de serviço.

Atualmente existem dois desafios relacionados ao desenvolvimento de instrumentos para avaliação do clima organizacional. O primeiro deles diz respeito à criação de itens que sejam sensíveis à mensuração de características particulares de cada realidade de trabaIho. Desta forma, devem-se considerar tanto aspectos macro-organizacionais, como o tipo de atividade reali- 
zada, o serviço oferecido pela organização e o setor que ela ocupa na economia, quanto elementos microorganizacionais, tal qual a formação profissional dos trabaIhadores e o modo como eles percebem e interpretam a cultura organizacional. Um segundo desafio reside justamente no polo oposto dessa necessidade inicial: desenvolver itens que sejam mais gerais e que possam avaliar contextos culturais cada vez mais amplos. Para tanto, é desejável que sejam concebidos itens que também possam ser testados em sujeitos de diferentes amostras e em organizações diversas, com o intuito de se verificar o poder de generalização dos itens e, consequentemente, para que se possam avaliar os possíveis limites da medida desenvolvida.

Ao buscar integrar os desafios supracitados, a Escala de Clima Organizacional para Organizações de Saúde é composta tanto de itens desenvolvidos para avaliar a especificidade da realidade de trabalho dos profissionais da organização de saúde investigada (a exemplo de alguns descritores específicos que medem aspectos de relacionamento com a comunidade), como de itens que mensuram características mais gerais (como liderança e desenvolvimento profissional). Assim, muitos dos itens desenvolvidos para avaliar o construto clima em organizações de saúde podem ser adaptados para outros contextos organizacionais, desde que sejam submetidos novamente ao exame da sua validade de construto, a fim de verificar se o modelo fatorial encontrado demonstra equivalência psicométrica com os resultados de amostras com perfis distintos. Vale ressaltar ainda que a escala validada a partir dessa pesquisa reflete características amostrais específicas, inerentes a setores organizacionais de saúde pertencentes a uma realidade social e cultural oportuna ao contexto investigado. Assim, é importante que se ampliem os estudos da estrutura fatorial da escala para outras organizações de saúde, em outras regiões geográficas.

Com o desenvolvimento e o estudo da validade de construto da Escala de Clima Organizacional para Organizações de Saúde buscou-se trazer contribuições teóricas significativas para a produção de conhecimento sobre a dimensionalidade do clima organizacional e a representatividade dos descritores na mensuração do atributo. Além disso, tal proposta teve por escopo fornecer evidências empíricas que garantam a devida utilização da ferramenta para diagnóstico organizacional em futuras pesquisas em organizações de saúde, além de oferecer contribuições que tornem possível o desenvolvimento de instrumentos de avaliação do clima organizacional para outras realidades de trabalho.

\section{Referências}

Argyris, C. (1958). Some problems in conceptualizing organizational climate: a case study of a bank. Administrative Science Quarterly, 2 (4), 501-520.

Brasil. Ministério da Saúde. (1996). Resolução 196/96 do Conselho Nacional de Saúde. Dispõe sobre a ética na pesquisa com seres humanos. Brasília: Ministério da Saúde.

Conselho Federal de Psicologia (2000). Resolução 0016/2000. Dispõe sobre a pesquisa em psicologia com seres humanos. Brasília: Conselho Federal de Psicologia.

Cooke, R. A., \& Lafferty, J. C. (1989). Organizational culture inventory. Plymouth, Ml: Human Synergistics.

Crespo, M. L. F. (2005). Construção e validação de um instrumento de clima para criatividade nas organizações empresariais. Tese de doutorado não-publicada, Pontifícia Universidade Católica de Campinas.

Forehand, G. A., \& Gilmer, H. B. (1964). Environmental variation in studies of organizational behavior. Psychological Bulletin, 62 (6), 361-382.

Friedlander, F., \& Margulies, N. (1969). Multiple impacts of organizational climate and individual value systems upon job satisfaction. Personnel Psychology, 22 (2), 171-183.

Glick, W. H. (1985). Conceptualizing and measuring organizational and psychological climate: pitfalls in multi-level research. Academy of Management Review, $10(3), 601-616$.

Glisson, C., \& James, L. R. (2002). The cross-level effects of culture and climate in human service teams. Journal of Organizational Behavior, 23 (6), 767-794.

Gorsuch, R. L. (1983). Factor analysis. New Jersey: Lawrence Erlbaum.

Hair, J. F. Jr., Anderson, R. E., Tatham, R. L., \& Black, W. C. (1998). Multivariate data analysis. New Jersey: Prentice Hall.

Hellriegel, D., \& Slocum, J. W. Jr. (1974). Organizational climate: measures, research and contingencies. Academy of Management Journal, 17 (2), 255-280.

Hofstede, G., Neuijen, B., Ohayv, D. D., \& Sanders, G. (1990). Measuring organizational cultures: a qualitative and quantitative study across twenty cases. Administrative Science Quarterly, 35 (2), 286-316.

House, R. J., \& Rizzo, J. R. (1972). Toward the measurement of organizational practices: scale development and validation. Journal of Applied Psychology, 56 (5), 388-396.

Kroeber, A. L., \& Kluckhohn, C. (1952). Culture: a critical review of concepts and definitions. New York: Vintage Books. 
Laros, J. A., \& Puente-Palacios, K. E. (2004). Validação cruzada de uma escala de clima social. Estudos de Psicologia (Natal), 9 (1), 113-119.

Lewin, K. (1939). Field theory and experiment in social psychology: concept and methods. American Journal of Sociology, 44 (6), 868-896.

Lewin, K., Lippitt, R., \&White, R. (1939). Patterns of aggressive behaviors in experimentally created social climates. Journal of Social Psychology, 10, 271-299.

Litwin, G. H., \& Stringer, R. A. (1968). Motivation and organizational climate. Cambridge: Harvard University Press.

Malhotra, N. K. (2001). Pesquisa de Marketing: uma orientação aplicada. Porto Alegre: Bookman.

Martins, M. C. F. (2000). Clima organizacional: o estado da arte. Revista da Sociedade de Psicologia do Triângulo Mineiro, 3, 12-18.

Martins, M. C. F., Oliveira, B., Silva, C. F., Pereira, K. C., \& Sousa, M. R. (2004). Construção e validação de uma escala de medida de clima organizacional. Psicologia: Organizações e Trabalho, 4 (1), 37-60.

Moran, E. T., \& Volkwein, J. F. (1992). The cultural approach to the formation of organizational climate. Human Relations, 45 (1), 19-47.

Ouchi, W. G. (1981). Theory Z: how American business can meet the Japanese challenge. Reading, MA: Addison-Wesley.

Pasquali, L. (1998). Princípios de elaboração de escalas psicológicas. Revista de Psiquiatria Clínica, 25 (5 Edição Especial), 206-213.

Pasquali, L. (1999). Histórico dos instrumentos psicológicos. In L. Pasquali (Org.), instrumentos psicológicos: manual prático de elaboração (pp.13-25). Brasília: Editora UnB.

Payne, R. L., \& Pheysey, D. C. (1971). G. C. Stern's organizational climate index: a reconceptualization and application to business organizations. Organizational Behavior and Human Performance, 6 (1), 120-125.

Pritchard, R. D., \& Karasick, B. W. (1973). The effects of organizational climate on managerial job performance and job satisfaction. Organizational Behavior and Human Performance, 9 (1), 126-146.

Schein, E. H. (1985). Organizational culture and leadership. San Francisco: Jossey-Bass.

Schneider, B., \& Bartlett, C. J. (1970). Individual differences and organizational climate $\|$ : measurement of organizational climate by the multi-trait, multi-rater matrix. Personnel Psychology, 23 (4), 493-512.

Schneider, B., \& Reichers, A. E. (1983). On etiology of climates. Personnel Psychology, 36 (1), 19-39.

Schneider, B., Salvaggio, A. N., \& Subirats, M. (2002). Climate strength: a new direction for climate research. Journal of Applied Psychology, 87 (2), 220-229.

Silva, N., \& Zanelli, J. C. (2004). Cultura Organizacional. In J. E. Borges-Andrade \& J. C. Zanelli (Orgs.), Psicologia, organizações e trabalho no Brasil (pp.407-442). Porto Alegre: Artmed.

Smith, G. T., \& McCarthy, D. M. (1995). Methodological considerations in the refinement of clinical assessment instruments. Psychological Assessment, 7 (3), 300-308.

Swartz, M., \& Jordan, D. (1980). Culture: an anthropological perspective. New York: Wiley.

Tabachnik, B. G., \& Fidell, L. S. (1996). Using multivariate statistics. New York: Harper \& Row.

Taguiri, R., \& Litwin, G. H. (1968). Organizational climate: exploration of a concept. London: Harvard University Press.

Tamayo, A. (1999). Valores e clima organizacional. In M. G. T Paz \& A. Tamayo (Orgs.), Escola, saúde e trabalho: estudos psicológicos (pp.261-289). Brasília: Editora da UnB.

Toro, F. A. (2001). El clima organizacional: perfil de empresas colombianas. Medellín: Cicel Ltda.

Trice, H. M., \& Beyer, J. M. (1984). Studying organizational cultures through rites and ceremonials. Academy of Management Review, 9 (4), 653-669.

Recebido em: 6/8/2007

Versão final reapresentada em: 24/4/2009

Aprovado em: 26/5/2009 